\title{
Can Academics Provide Value to Practitioners? The Practitioner Response to Academic Research Output: An Abstract
}

\author{
Christian Hinsch, Joseph Horak, and Josip Kotlar
}

\begin{abstract}
Ten years have passed since Van de Ven and Johnson published their original Academy of Management Review article on engaged scholarship. With over 1,000 citations, this article has gained significant attention. Yet questions remain as to whether the behavior of the academic community has truly changed in a manner that engages business community stakeholders with respect to creating customer value through the co-creation of knowledge. While the engaged scholarship approach faces many obstacles within academia, the current study explores the external obstacles, specifically the degree to which top managers utilize published research along with how manager's attitudes about research impact this process. In addition, this research explores business leader's perceptions about what academics do and what they should do from the practitioners' perspective.

Positioning practitioners as the consumers of academic output, the current paper explores the gap between academic knowledge and practitioner knowledge, and this analysis shows evidence of a practitioner dissatisfaction effect. In addition, practitioners perceive practitioner and academic knowledge to be distinct forms of knowing. However, there is also evidence of a knowledge transfer problem in the mediated relationships between business press usage, applied research usage, and academic research usage. This mediated effect is moderated by both practitioner expectations of academics and practitioner perceptions of academics.
\end{abstract}

References Available Upon Request

\author{
C. Hinsch $(\square)$ \\ Grand Valley State University, Grand Rapids, MI, USA \\ e-mail: hinschc@gvsu.edu \\ J. Horak \\ Horak Family Psychological Services, Grand Rapids, MI, USA \\ e-mail: joe@drhorak.com \\ J. Kotlar \\ Lancaster University, Lancaster, UK \\ e-mail: kotlar@lancaster.ac.uk
}

